BABAR-CONF-04/006

SLAC-PUB-10642

hep-ex/0408052

August 2004

\title{
A study of $\bar{B}^{0} \rightarrow D^{(*) 0} \bar{K}^{(*) 0}$ decays
}

\author{
The BABAR Collaboration
}

August 13, 2004

\begin{abstract}
We present a study of the decays $\bar{B}^{0} \rightarrow D^{(*) 0} \bar{K}^{(*) 0}$ using a sample of 124 million $\Upsilon(4 S) \rightarrow B \bar{B}$ decays collected with the BABAR detector at the PEP-II asymmetric-energy $e^{+} e^{-}$collider at SLAC. We report evidence for the decay of $B^{0}$ and $\bar{B}^{0}$ mesons to the $D^{* 0} K_{S}^{0}$ final state with an average branching fraction $\mathcal{B}\left(B \rightarrow D^{* 0} \bar{K}^{0}\right)=(4.5 \pm 1.9 \pm 0.5) \times 10^{-5}$. Similarly, we measure $\mathcal{B}(B \rightarrow$ $\left.D^{0} \bar{K}^{0}\right)=(6.2 \pm 1.2 \pm 0.4) \times 10^{-5}$ for the $D^{0} K_{S}^{0}$ final state. We also measure $\mathcal{B}\left(\bar{B}^{0} \rightarrow D^{0} \bar{K}^{* 0}\right)=$ $(6.2 \pm 1.4 \pm 0.6) \times 10^{-5}$ and set a $90 \%$ C.L. upper limit $\mathcal{B}\left(\bar{B}^{0} \rightarrow \bar{D}^{0} \bar{K}^{* 0}\right)<4.1 \times 10^{-5}$. All results presented in this paper are preliminary.
\end{abstract}

Submitted to the $32^{\text {nd }}$ International Conference on High-Energy Physics, ICHEP 04, 16 August - 22 August 2004, Beijing, China

Stanford Linear Accelerator Center, Stanford University, Stanford, CA 94309

Work supported in part by Department of Energy contract DE-AC03-76SF00515. 
The BABAR Collaboration,

B. Aubert, R. Barate, D. Boutigny, F. Couderc, J.-M. Gaillard, A. Hicheur, Y. Karyotakis, J. P. Lees, V. Tisserand, A. Zghiche

Laboratoire de Physique des Particules, F-74941 Annecy-le-Vieux, France

A. Palano, A. Pompili

Università di Bari, Dipartimento di Fisica and INFN, I-70126 Bari, Italy

J. C. Chen, N. D. Qi, G. Rong, P. Wang, Y. S. Zhu

Institute of High Energy Physics, Beijing 100039, China

G. Eigen, I. Ofte, B. Stugu

University of Bergen, Inst. of Physics, N-5007 Bergen, Norway

G. S. Abrams, A. W. Borgland, A. B. Breon, D. N. Brown, J. Button-Shafer, R. N. Cahn, E. Charles, C. T. Day, M. S. Gill, A. V. Gritsan, Y. Groysman, R. G. Jacobsen, R. W. Kadel, J. Kadyk, L. T. Kerth, Yu. G. Kolomensky, G. Kukartsev, G. Lynch, L. M. Mir, P. J. Oddone, T. J. Orimoto, M. Pripstein, N. A. Roe, M. T. Ronan, V. G. Shelkov, W. A. Wenzel

Lawrence Berkeley National Laboratory and University of California, Berkeley, CA 94720, USA

M. Barrett, K. E. Ford, T. J. Harrison, A. J. Hart, C. M. Hawkes, S. E. Morgan, A. T. Watson University of Birmingham, Birmingham, B15 2TT, United Kingdom

M. Fritsch, K. Goetzen, T. Held, H. Koch, B. Lewandowski, M. Pelizaeus, M. Steinke Ruhr Universität Bochum, Institut für Experimentalphysik 1, D-44780 Bochum, Germany

J. T. Boyd, N. Chevalier, W. N. Cottingham, M. P. Kelly, T. E. Latham, F. F. Wilson University of Bristol, Bristol BS8 1TL, United Kingdom

T. Cuhadar-Donszelmann, C. Hearty, N. S. Knecht, T. S. Mattison, J. A. McKenna, D. Thiessen University of British Columbia, Vancouver, BC, Canada V6T $1 Z 1$

A. Khan, P. Kyberd, L. Teodorescu Brunel University, Uxbridge, Middlesex UB8 3PH, United Kingdom

A. E. Blinov, V. E. Blinov, V. P. Druzhinin, V. B. Golubev, V. N. Ivanchenko, E. A. Kravchenko, A. P. Onuchin, S. I. Serednyakov, Yu. I. Skovpen, E. P. Solodov, A. N. Yushkov

Budker Institute of Nuclear Physics, Novosibirsk 630090, Russia

D. Best, M. Bruinsma, M. Chao, I. Eschrich, D. Kirkby, A. J. Lankford, M. Mandelkern, R. K. Mommsen, W. Roethel, D. P. Stoker University of California at Irvine, Irvine, CA 92697, USA

C. Buchanan, B. L. Hartfiel

University of California at Los Angeles, Los Angeles, CA 90024, USA

S. D. Foulkes, J. W. Gary, B. C. Shen, K. Wang

University of California at Riverside, Riverside, CA 92521, USA 
D. del Re, H. K. Hadavand, E. J. Hill, D. B. MacFarlane, H. P. Paar, Sh. Rahatlou, V. Sharma University of California at San Diego, La Jolla, CA 92093, USA

J. W. Berryhill, C. Campagnari, B. Dahmes, O. Long, A. Lu, M. A. Mazur, J. D. Richman, W. Verkerke University of California at Santa Barbara, Santa Barbara, CA 93106, USA

T. W. Beck, A. M. Eisner, C. A. Heusch, J. Kroseberg, W. S. Lockman, G. Nesom, T. Schalk, B. A. Schumm, A. Seiden, P. Spradlin, D. C. Williams, M. G. Wilson

University of California at Santa Cruz, Institute for Particle Physics, Santa Cruz, CA 95064, USA

J. Albert, E. Chen, G. P. Dubois-Felsmann, A. Dvoretskii, D. G. Hitlin, I. Narsky, T. Piatenko, F. C. Porter, A. Ryd, A. Samuel, S. Yang

California Institute of Technology, Pasadena, CA 91125, USA

S. Jayatilleke, G. Mancinelli, B. T. Meadows, M. D. Sokoloff

University of Cincinnati, Cincinnati, OH 45221, USA

T. Abe, F. Blanc, P. Bloom, S. Chen, W. T. Ford, U. Nauenberg, A. Olivas, P. Rankin, J. G. Smith, J. Zhang, L. Zhang University of Colorado, Boulder, CO 80309, USA

A. Chen, J. L. Harton, A. Soffer, W. H. Toki, R. J. Wilson, Q. Zeng Colorado State University, Fort Collins, CO 80523, USA

D. Altenburg, T. Brandt, J. Brose, M. Dickopp, E. Feltresi, A. Hauke, H. M. Lacker, R. Müller-Pfefferkorn, R. Nogowski, S. Otto, A. Petzold, J. Schubert, K. R. Schubert, R. Schwierz, B. Spaan, J. E. Sundermann

Technische Universität Dresden, Institut für Kern- und Teilchenphysik, D-01062 Dresden, Germany

D. Bernard, G. R. Bonneaud, F. Brochard, P. Grenier, S. Schrenk, Ch. Thiebaux, G. Vasileiadis, M. Verderi Ecole Polytechnique, LLR, F-91128 Palaiseau, France

D. J. Bard, P. J. Clark, D. Lavin, F. Muheim, S. Playfer, Y. Xie

University of Edinburgh, Edinburgh EH9 3JZ, United Kingdom

M. Andreotti, V. Azzolini, D. Bettoni, C. Bozzi, R. Calabrese, G. Cibinetto, E. Luppi, M. Negrini, L. Piemontese, A. Sarti

Università di Ferrara, Dipartimento di Fisica and INFN, I-44100 Ferrara, Italy

E. Treadwell

Florida A $6 M$ University, Tallahassee, FL 3230\%, USA

F. Anulli, R. Baldini-Ferroli, A. Calcaterra, R. de Sangro, G. Finocchiaro, P. Patteri, I. M. Peruzzi, M. Piccolo, A. Zallo

Laboratori Nazionali di Frascati dell'INFN, I-00044 Frascati, Italy

A. Buzzo, R. Capra, R. Contri, G. Crosetti, M. Lo Vetere, M. Macri, M. R. Monge, S. Passaggio, C. Patrignani, E. Robutti, A. Santroni, S. Tosi

Università di Genova, Dipartimento di Fisica and INFN, I-16146 Genova, Italy

S. Bailey, G. Brandenburg, K. S. Chaisanguanthum, M. Morii, E. Won

Harvard University, Cambridge, MA 02138, USA 
R. S. Dubitzky, U. Langenegger

Universität Heidelberg, Physikalisches Institut, Philosophenweg 12, D-69120 Heidelberg, Germany

W. Bhimji, D. A. Bowerman, P. D. Dauncey, U. Egede, J. R. Gaillard, G. W. Morton, J. A. Nash, M. B. Nikolich, G. P. Taylor Imperial College London, London, SWr 2AZ, United Kingdom

M. J. Charles, G. J. Grenier, U. Mallik

University of Iowa, Iowa City, IA 52242, USA

J. Cochran, H. B. Crawley, J. Lamsa, W. T. Meyer, S. Prell, E. I. Rosenberg, A. E. Rubin, J. Yi Iowa State University, Ames, IA 50011-3160, USA

M. Biasini, R. Covarelli, M. Pioppi

Università di Perugia, Dipartimento di Fisica and INFN, I-06100 Perugia, Italy

M. Davier, X. Giroux, G. Grosdidier, A. Höcker, S. Laplace, F. Le Diberder, V. Lepeltier, A. M. Lutz, T. C. Petersen, S. Plaszczynski, M. H. Schune, L. Tantot, G. Wormser

Laboratoire de l'Accélérateur Linéaire, F-91898 Orsay, France

C. H. Cheng, D. J. Lange, M. C. Simani, D. M. Wright

Lawrence Livermore National Laboratory, Livermore, CA 94550, USA

A. J. Bevan, C. A. Chavez, J. P. Coleman, I. J. Forster, J. R. Fry, E. Gabathuler, R. Gamet, D. E. Hutchcroft, R. J. Parry, D. J. Payne, R. J. Sloane, C. Touramanis

University of Liverpool, Liverpool L69 72E, United Kingdom

J. J. Back, ${ }^{1}$ C. M. Cormack, P. F. Harrison, ${ }^{1}$ F. Di Lodovico, G. B. Mohanty ${ }^{1}$

Queen Mary, University of London, E1 4NS, United Kingdom

C. L. Brown, G. Cowan, R. L. Flack, H. U. Flaecher, M. G. Green, P. S. Jackson, T. R. McMahon, S. Ricciardi, F. Salvatore, M. A. Winter

University of London, Royal Holloway and Bedford New College, Egham, Surrey TW20 0EX, United Kingdom

D. Brown, C. L. Davis

University of Louisville, Louisville, KY 40292, USA

J. Allison, N. R. Barlow, R. J. Barlow, P. A. Hart, M. C. Hodgkinson, G. D. Lafferty, A. J. Lyon, J. C. Williams

University of Manchester, Manchester M13 9PL, United Kingdom

A. Farbin, W. D. Hulsbergen, A. Jawahery, D. Kovalskyi, C. K. Lae, V. Lillard, D. A. Roberts

University of Maryland, College Park, MD 20742, USA

G. Blaylock, C. Dallapiccola, K. T. Flood, S. S. Hertzbach, R. Kofler, V. B. Koptchev, T. B. Moore, S. Saremi, H. Staengle, S. Willocq

University of Massachusetts, Amherst, MA 01003, USA

\footnotetext{
${ }^{1}$ Now at Department of Physics, University of Warwick, Coventry, United Kingdom
} 
R. Cowan, G. Sciolla, S. J. Sekula, F. Taylor, R. K. Yamamoto

Massachusetts Institute of Technology, Laboratory for Nuclear Science, Cambridge, MA 02139, USA

D. J. J. Mangeol, P. M. Patel, S. H. Robertson

McGill University, Montréal, QC, Canada H3A 2T8

A. Lazzaro, V. Lombardo, F. Palombo

Università di Milano, Dipartimento di Fisica and INFN, I-20133 Milano, Italy

J. M. Bauer, L. Cremaldi, V. Eschenburg, R. Godang, R. Kroeger, J. Reidy, D. A. Sanders, D. J. Summers, H. W. Zhao

University of Mississippi, University, MS 38677, USA

S. Brunet, D. Côté, P. Taras

Université de Montréal, Laboratoire René J. A. Lévesque, Montréal, QC, Canada H3C 3J7

H. Nicholson

Mount Holyoke College, South Hadley, MA 01075, USA

N. Cavallo, ${ }^{2}$ F. Fabozzi, ${ }^{2}$ C. Gatto, L. Lista, D. Monorchio, P. Paolucci, D. Piccolo, C. Sciacca

Università di Napoli Federico II, Dipartimento di Scienze Fisiche and INFN, I-80126, Napoli, Italy

M. Baak, H. Bulten, G. Raven, H. L. Snoek, L. Wilden

NIKHEF, National Institute for Nuclear Physics and High Energy Physics, NL-1009 DB Amsterdam, The Netherlands

C. P. Jessop, J. M. LoSecco

University of Notre Dame, Notre Dame, IN 46556, USA

T. Allmendinger, K. K. Gan, K. Honscheid, D. Hufnagel, H. Kagan, R. Kass, T. Pulliam, A. M. Rahimi, R. Ter-Antonyan, Q. K. Wong

Ohio State University, Columbus, OH 43210, USA

J. Brau, R. Frey, O. Igonkina, C. T. Potter, N. B. Sinev, D. Strom, E. Torrence

University of Oregon, Eugene, OR 97403, USA

F. Colecchia, A. Dorigo, F. Galeazzi, M. Margoni, M. Morandin, M. Posocco, M. Rotondo, F. Simonetto, R. Stroili, G. Tiozzo, C. Voci

Università di Padova, Dipartimento di Fisica and INFN, I-35131 Padova, Italy

M. Benayoun, H. Briand, J. Chauveau, P. David, Ch. de la Vaissière, L. Del Buono, O. Hamon, M. J. J. John, Ph. Leruste, J. Malcles, J. Ocariz, M. Pivk, L. Roos, S. T'Jampens, G. Therin Universités Paris VI et VII, Laboratoire de Physique Nucléaire et de Hautes Energies, F-75252 Paris, France

P. F. Manfredi, V. Re

Università di Pavia, Dipartimento di Elettronica and INFN, I-27100 Pavia, Italy

\footnotetext{
${ }^{2}$ Also with Università della Basilicata, Potenza, Italy
} 


\author{
P. K. Behera, L. Gladney, Q. H. Guo, J. Panetta \\ University of Pennsylvania, Philadelphia, PA 19104, USA
}

C. Angelini, G. Batignani, S. Bettarini, M. Bondioli, F. Bucci, G. Calderini, M. Carpinelli, F. Forti, M. A. Giorgi, A. Lusiani, G. Marchiori, F. Martinez-Vidal, ${ }^{3}$ M. Morganti, N. Neri, E. Paoloni, M. Rama, G. Rizzo, F. Sandrelli, J. Walsh

Università di Pisa, Dipartimento di Fisica, Scuola Normale Superiore and INFN, I-56127 Pisa, Italy

M. Haire, D. Judd, K. Paick, D. E. Wagoner

Prairie View A\&M University, Prairie View, TX 77446, USA

N. Danielson, P. Elmer, Y. P. Lau, C. Lu, V. Miftakov, J. Olsen, A. J. S. Smith, A. V. Telnov

Princeton University, Princeton, NJ 08544, USA

F. Bellini, G. Cavoto, ${ }^{4}$ R. Faccini, F. Ferrarotto, F. Ferroni, M. Gaspero, L. Li Gioi, M. A. Mazzoni, S. Morganti, M. Pierini, G. Piredda, F. Safai Tehrani, C. Voena Università di Roma La Sapienza, Dipartimento di Fisica and INFN, I-00185 Roma, Italy

S. Christ, G. Wagner, R. Waldi

Universität Rostock, D-18051 Rostock, Germany

T. Adye, N. De Groot, B. Franek, N. I. Geddes, G. P. Gopal, E. O. Olaiya

Rutherford Appleton Laboratory, Chilton, Didcot, Oxon, OX11 0QX, United Kingdom

R. Aleksan, S. Emery, A. Gaidot, S. F. Ganzhur, P.-F. Giraud, G. Hamel de Monchenault, W. Kozanecki, M. Legendre, G. W. London, B. Mayer, G. Schott, G. Vasseur, Ch. Yèche, M. Zito

DSM/Dapnia, CEA/Saclay, F-91191 Gif-sur-Yvette, France

M. V. Purohit, A. W. Weidemann, J. R. Wilson, F. X. Yumiceva

University of South Carolina, Columbia, SC 29208, USA

D. Aston, R. Bartoldus, N. Berger, A. M. Boyarski, O. L. Buchmueller, R. Claus, M. R. Convery, M. Cristinziani, G. De Nardo, D. Dong, J. Dorfan, D. Dujmic, W. Dunwoodie, E. E. Elsen, S. Fan,

R. C. Field, T. Glanzman, S. J. Gowdy, T. Hadig, V. Halyo, C. Hast, T. Hryn'ova, W. R. Innes,

M. H. Kelsey, P. Kim, M. L. Kocian, D. W. G. S. Leith, J. Libby, S. Luitz, V. Luth, H. L. Lynch,

H. Marsiske, R. Messner, D. R. Muller, C. P. O'Grady, V. E. Ozcan, A. Perazzo, M. Perl, S. Petrak,

B. N. Ratcliff, A. Roodman, A. A. Salnikov, R. H. Schindler, J. Schwiening, G. Simi, A. Snyder, A. Soha, J. Stelzer, D. Su, M. K. Sullivan, J. Va'vra, S. R. Wagner, M. Weaver, A. J. R. Weinstein,

W. J. Wisniewski, M. Wittgen, D. H. Wright, A. K. Yarritu, C. C. Young

Stanford Linear Accelerator Center, Stanford, CA 94309, USA

P. R. Burchat, A. J. Edwards, T. I. Meyer, B. A. Petersen, C. Roat

Stanford University, Stanford, CA 94305-4060, USA

S. Ahmed, M. S. Alam, J. A. Ernst, M. A. Saeed, M. Saleem, F. R. Wappler

State University of New York, Albany, NY 12222, USA

\footnotetext{
${ }^{3}$ Also with IFIC, Instituto de Física Corpuscular, CSIC-Universidad de Valencia, Valencia, Spain

${ }^{4}$ Also with Princeton University, Princeton, USA
} 
W. Bugg, M. Krishnamurthy, S. M. Spanier

University of Tennessee, Knoxville, TN 37996, USA

R. Eckmann, H. Kim, J. L. Ritchie, A. Satpathy, R. F. Schwitters

University of Texas at Austin, Austin, TX 78712, USA

J. M. Izen, I. Kitayama, X. C. Lou, S. Ye

University of Texas at Dallas, Richardson, TX 75083, USA

F. Bianchi, M. Bona, F. Gallo, D. Gamba

Università di Torino, Dipartimento di Fisica Sperimentale and INFN, I-10125 Torino, Italy

L. Bosisio, C. Cartaro, F. Cossutti, G. Della Ricca, S. Dittongo, S. Grancagnolo, L. Lanceri, P. Poropat, ${ }^{5}$ L. Vitale, G. Vuagnin

Università di Trieste, Dipartimento di Fisica and INFN, I-34127 Trieste, Italy

R. S. Panvini

Vanderbilt University, Nashville, TN 37235, USA

Sw. Banerjee, C. M. Brown, D. Fortin, P. D. Jackson, R. Kowalewski, J. M. Roney, R. J. Sobie University of Victoria, Victoria, BC, Canada V8W $3 P 6$

H. R. Band, B. Cheng, S. Dasu, M. Datta, A. M. Eichenbaum, M. Graham, J. J. Hollar, J. R. Johnson, P. E. Kutter, H. Li, R. Liu, A. Mihalyi, A. K. Mohapatra, Y. Pan, R. Prepost, P. Tan, J. H. von Wimmersperg-Toeller, J. Wu, S. L. Wu, Z. Yu

University of Wisconsin, Madison, WI 53706, USA

M. G. Greene, H. Neal

Yale University, New Haven, CT 06511, USA

\footnotetext{
${ }^{5}$ Deceased
} 
With the discovery of $C P$ violation in the decays of neutral $B$ mesons [1] and the precise measurement [2] of the angle $\beta$ of the Cabibbo-Kobayashi-Maskawa (CKM) Unitarity Triangle [3], the experimental focus has shifted toward the measurements of the angles $\alpha$ and $\gamma$. Several methods have been suggested to measure $\gamma$ with small uncertainties, but they all require large samples of $B$ mesons not yet available. The decay modes $\bar{B}^{0} \rightarrow D^{(*) 0} \bar{K}^{0}$ offer a new approach for determination of $\sin (2 \beta+\gamma)$ from measurement of time-dependent $C P$ asymmetries in these decays [4]. The $C P$ asymmetry appears as a result of interference between two possible diagrams leading to the same final state $D^{(*) 0} K_{S}^{0}$ (Figure 1): a $b \rightarrow c$ transition $\bar{B}^{0} \rightarrow D^{(*) 0} K^{0}$ and a $\bar{b} \rightarrow \bar{u}$ transition $B^{0} \rightarrow D^{(*) 0} K^{0}$. A $\bar{B}^{0}$ meson can either decay to a $D^{(*) 0} \bar{K}^{0}\left(\bar{D}^{(*) 0} \bar{K}^{0}\right)$ final state, or oscillate into a $B^{0}$ which then decays to a $D^{(*) 0} K^{0}\left(\bar{D}^{(*) 0} K^{0}\right)$ final state.

The $\bar{B}^{0} B^{0}$ oscillation provides the weak phase $2 \beta$ and the relative phase of the two decay diagrams is $\gamma$.
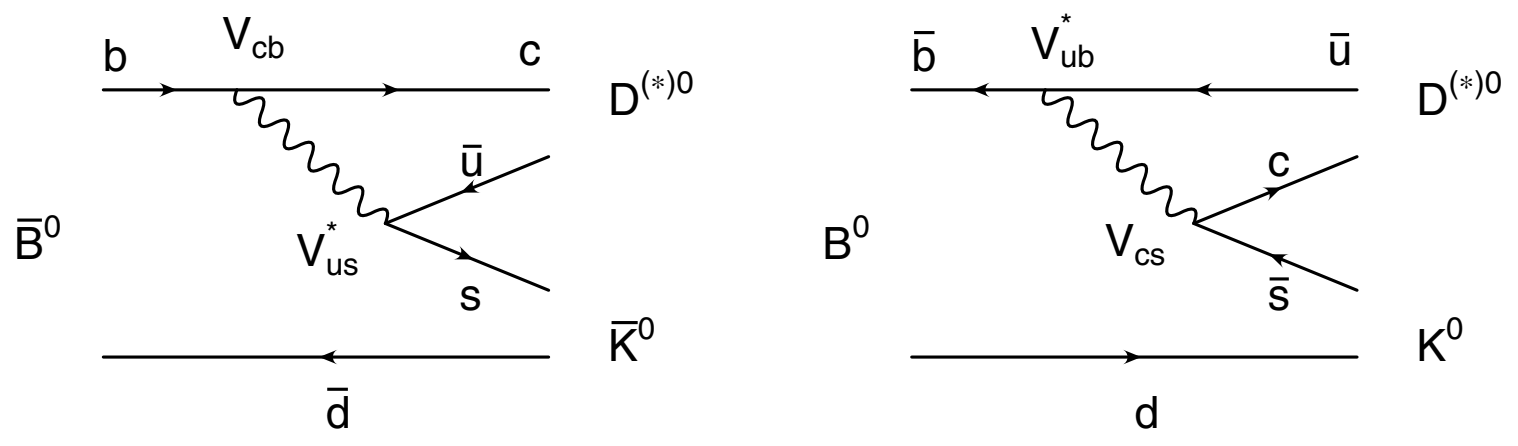

Figure 1: The decay diagrams for the $b \rightarrow c$ transition $\bar{B}^{0} \rightarrow D^{(*) 0} \bar{K}^{0}$ and the $\bar{b} \rightarrow \bar{u}$ transition $B^{0} \rightarrow D^{(*) 0} K^{0}$.

The sensitivity of this method depends on the ratio $r \equiv\left|\mathcal{A}\left(\bar{B}^{0} \rightarrow \bar{D}^{(*) 0} \bar{K}^{0}\right)\right| / \mid \mathcal{A}\left(\bar{B}^{0} \rightarrow\right.$ $\left.D^{(*) 0} \bar{K}^{0}\right) \mid[4]$ of the decay amplitudes. The ratio of the CKM matrix elements in the two amplitudes naively suggests $r \approx 0.4$, however, the $B$ decay dynamics can modify this expectation. The ratio $r$ can be probed by measuring the rate for the decays $\bar{B}^{0} \rightarrow D^{0} \bar{K}^{* 0}$ and $\bar{B}^{0} \rightarrow \bar{D}^{0} \bar{K}^{* 0}$, using the self-tagging decay $\bar{K}^{* 0} \rightarrow K^{-} \pi^{+}$.

The $\bar{B}^{0} \rightarrow D^{0} \bar{K}^{* 0}$ and $\bar{B}^{0} \rightarrow \bar{D}^{0} \bar{K}^{* 0}$ decays are separated by means of the correlation between the charge of the kaons produced in the $D^{0}$ and $K^{* 0}$ decays: in the former decay the two kaons must have the same charge, while in the latter they are oppositely charged. In the $\bar{B}^{0} \rightarrow D^{(*) 0} \bar{K}^{0}$ decays, the strangeness content of the $\bar{K}^{0}$ is hidden and one cannot distinguish between $\bar{B}^{0} \rightarrow D^{(*) 0} \bar{K}^{0}$ and $\bar{B}^{0} \rightarrow \bar{D}^{(*) 0} \bar{K}^{0}$. Hence in the remainder of this paper we refer to these decays as $B \rightarrow D^{(*) 0} \bar{K}^{0}$. We estimate the branching fractions $\mathcal{B}\left(B \rightarrow D^{(*) 0} \bar{K}^{(*) 0}\right)$ from the measured color-suppressed decays $\bar{B}^{0} \rightarrow D^{(*) 0} \pi^{0}[5]$ to be approximately $3 \times 10^{-5}$. In this paper, we present the first evidence for the decay $B \rightarrow D^{* 0} \bar{K}^{0}$, measurements of the branching fractions $\mathcal{B}\left(B \rightarrow D^{0} \bar{K}^{0}\right)$ and $\mathcal{B}\left(\bar{B}^{0} \rightarrow D^{0} \bar{K}^{* 0}\right)$, and a $90 \%$ C.L. upper limit for the branching fraction of the $b \rightarrow u$ transition $\bar{B}^{0} \rightarrow \bar{D}^{0} \bar{K}^{* 0}$.

Results presented here are based on a sample of 124 million $\Upsilon(4 S) \rightarrow B \bar{B}$ decays collected with the BABAR detector between 1999 and 2003 at the PEP-II asymmetric-energy $e^{+} e^{-}$collider operating near the $\Upsilon(4 S)$ resonance. The properties of the continuum $e^{+} e^{-} \rightarrow q \bar{q}(q=u, d, s, c)$ events are studied with a data sample of $12 \mathrm{fb}^{-1}$ recorded $40 \mathrm{MeV}$ below the $\Upsilon(4 S)$ resonance. We also use large samples of simulated $\Upsilon(4 S) \rightarrow B \bar{B}$ and $e^{+} e^{-} \rightarrow q \bar{q}$ events which are about three 
and 15 times the size of the data, respectively.

The BABAR detector is described elsewhere [6]. Only detector components relevant for this analysis are summarized here. Trajectories of charged particles are measured in a spectrometer consisting of a five-layer silicon vertex tracker (SVT) and a 40-layer drift chamber (DCH) operating in a $1.5 \mathrm{~T}$ axial magnetic field. Charged particles are identified as pions or kaons using information from a detector of internally reflected Cherenkov light, as well as measurements of energy loss from ionization $(d E / d x)$ in the SVT and the DCH. Photons are detected using an electromagnetic calorimeter (EMC) constructed of 6580 thallium-doped CsI crystals.

We reconstruct the decays $\bar{B}^{0} \rightarrow D^{0} \bar{K}^{0}, D^{* 0} \bar{K}^{0}, D^{0} \bar{K}^{* 0}$, and $\bar{D}^{0} \bar{K}^{* 0}$ in the decay chains: $D^{* 0} \rightarrow D^{0} \pi^{0} ; D^{0} \rightarrow K^{-} \pi^{+}, K^{-} \pi^{+} \pi^{0}$, and $K^{-} \pi^{+} \pi^{-} \pi^{+} ; K^{0} \rightarrow K_{S}^{0} \rightarrow \pi^{+} \pi^{-} ; K^{* 0} \rightarrow K^{+} \pi^{-}$; and $\pi^{0} \rightarrow \gamma \gamma$ (throughout this paper charge-conjugated decay modes are implied unless explicitly specified). For each decay channel, the optimal selection criteria are determined by maximizing the ratio $S / \sqrt{S+B}$, where $\mathrm{S}$ and $\mathrm{B}$ are, respectively, the estimated signal and background yields in simulated events. A large sample of the more abundant $B^{+} \rightarrow \bar{D}^{0} \pi^{+}$decays, in which the $\bar{D}^{0}$ decays to the $K^{+} \pi^{-}, K^{+} \pi^{-} \pi^{0}$, and $K^{+} \pi^{-} \pi^{+} \pi^{-}$final states, is used as a calibration sample to measure efficiencies and resolutions for the selection variables.

Charged tracks used to reconstruct $D^{0}$ and $K^{* 0}$ candidates are required to have transverse momentum $p_{T}>100 \mathrm{MeV} / c$, and the $K^{ \pm}$candidates must satisfy kaon identification criteria. These identification criteria have an average efficiency of about $90 \%$ while the probability of pions being mis-identified as kaons varies between a few percent and 15\%. The photons are reconstructed from clusters in the electromagnetic calorimeter with energy greater than $30 \mathrm{MeV}$ that are consistent with photon showers. We select $\pi^{0}$ candidates from pairs of photon candidates and require $115 \mathrm{MeV} / c^{2}<$ $m(\gamma \gamma)<150 \mathrm{MeV} / \mathrm{c}^{2}$.

The $K_{S}^{0}$ candidates are selected from pairs of oppositely charged tracks with invariant mass within $7 \mathrm{MeV} / c^{2}(2 \sigma)$ of the nominal $K_{S}^{0}$ mass [7]. The displacement $l_{K_{S}^{0}}$ of the $K_{S}^{0}$ decay vertex from the interaction point in the plane perpendicular to the beam axis divided by its measured uncertainty $\sigma_{K_{S}^{0}}$ must be greater than 2 . The $K^{* 0}$ candidates are selected from pairs of oppositely charged $K^{+}$and $\pi^{-}$tracks, with invariant mass $m\left(K^{+} \pi^{-}\right)$within $50 \mathrm{MeV} / c^{2}$ of the nominal $K^{* 0}$ mass [7]. We also require $\left|\cos \theta_{h}\right|>0.4$, where $\theta_{h}$ is the $K^{* 0}$ helicity angle, defined as the angle between the direction of the $K^{* 0}$ in the $B^{0}$-meson rest frame and the direction of its daughter $K^{+}$ in the $K^{* 0}$ rest frame. For signal $\bar{B}^{0} \rightarrow D^{0} \bar{K}^{* 0}$ candidates, $\theta_{h}$ follows a $\cos ^{2} \theta_{h}$ distribution while the combinatorial background is distributed uniformly.

We reconstruct the $D^{0}$ candidates in the $K^{-} \pi^{+}$and $K^{-} \pi^{+} \pi^{-} \pi^{+}$decay modes by combining charged tracks. Combinations with an invariant mass within $2 \sigma$ of the nominal $D^{0}$ mass $m_{D^{0}}$ are retained. In the $D^{0} \rightarrow K^{-} \pi^{+} \pi^{0}$ selection, the $\pi^{0}$ candidates are required to have a center-ofmass $(\mathrm{CM})$ momentum $p_{\pi^{0}}^{*}$ greater than $400 \mathrm{MeV} / c$. For each $K^{-} \pi^{+} \pi^{0}$ combination, we use the kinematics of the decay products and the known properties of the Dalitz plot for this decay [8] to compute the square of the decay amplitude $|A|^{2}$. We select combinations with $|A|^{2}$ greater than $5 \%$ of its maximum value. This requirement selects mostly the $K \rho$ region of the Dalitz plot. It rejects $62 \%$ of the combinatorial background and has an efficiency of $76 \%$, as measured with the $B^{+} \rightarrow \bar{D}^{0} \pi^{+}$calibration sample. The combinations with the invariant mass within $25 \mathrm{MeV} / c^{2}(2.5 \sigma)$ of $m_{D^{0}}$ are retained. For the purpose of cross checks, we define a $D^{0}$ mass sideband as the region between 45 and $90 \mathrm{MeV} / c^{2}$ away from $m_{D^{0}}$ in the $D^{0} \rightarrow K^{-} \pi^{+}$and $K^{-} \pi^{+} \pi^{-} \pi^{+}$mode, and between 85 and $160 \mathrm{MeV} / c^{2}$ away from $m_{D^{0}}$ in $D^{0} \rightarrow K^{-} \pi^{+} \pi^{0}$.

The $D^{* 0}$ candidates are selected from combinations of a $D^{0}$ and a $\pi^{0}$ with $p_{\pi^{0}}^{*}>70 \mathrm{MeV} / c$. After kinematically constraining $D^{0}$ and $\pi^{0}$ candidates to their nominal masses, we select the 
candidates with the mass difference $\Delta m \equiv\left|m\left(D^{* 0}\right)-m\left(D^{0}\right)-142.2 \mathrm{MeV} / c^{2}\right|<3.3 \mathrm{MeV} / c^{2}$.

Two standard kinematic variables are used to select $B^{0}$ candidates: the energy-substituted mass $m_{\mathrm{ES}} \equiv \sqrt{\left(\frac{1}{2} s+\mathbf{p}_{0} \cdot \mathbf{p}_{B}\right)^{2} / E_{0}^{2}-\mathbf{p}_{B}^{2}}$ and the energy difference $\Delta E \equiv E_{B}^{*}-\frac{1}{2} \sqrt{s}$, where the asterisk denotes the $\mathrm{CM}$ frame, $s$ is the square of the total energy in the CM frame, $\mathbf{p}$ and $E$ are, respectively, three-momentum and energy, and the subscripts 0 and $B$ refer to $\Upsilon(4 S)$ and $B^{0}$, respectively. For signal events, $m_{\mathrm{ES}}$ is centered around the $B^{0}$ mass with an r.m.s. resolution of about $2.6 \mathrm{MeV} / c^{2}$, dominated by the knowledge of the $e^{+}$and $e^{-}$beam energies. The $\Delta E$ resolution is dominated by the momentum and energy resolutions of the detector and hence varies for different decay modes. We constrain the mass of the $D^{(*) 0}$ and $K_{S}^{0}$ candidates to their respective nominal values. In simulated events the r.m.s. $\Delta E$ resolution is found to be $\approx 13 \mathrm{MeV}$ for all $B^{0}$ decay modes. The $B^{0}$ candidates are required to have $\Delta E$ within $30 \mathrm{MeV}$ of the mean value measured in the $B^{+} \rightarrow \bar{D}^{0} \pi^{+}$calibration sample.

We use two variables to reject most of the remaining background, which is dominated by continuum events: the polar angle $\theta_{B}^{*}$ of the $B^{0}$ candidate in the CM frame and a Fisher discriminant [9] based on the energy flow in the rest of the event, after removing the $B^{0}$ decay products. The Fisher discriminant is defined as a linear combination of $\left|\cos \theta_{T B}^{*}\right|$, where $\theta_{T B}^{*}$ is the angle in the CM frame between the thrust axis of the $B^{0}$ and that of the remaining charged and neutral particles in the rest of the event, and the Legendre monomials $\mathcal{L}^{0}$ and $\mathcal{L}^{2}$ defined as $\mathcal{L}^{i} \equiv \sum_{j} p_{j}^{*} \cos ^{i} \theta_{j}$; here $p_{j}^{*}$ is the CM momentum and $\theta_{j}$ the angle between the direction of remaining particles in the event with respect to the thrust axis of the $B^{0}$ candidate. The requirement on $\mathcal{F}$ varies for each decay channel because of different levels of the background. In the $D^{(*) 0} K_{S}^{0}$ and $D^{0} \bar{K}^{* 0}$ final states our requirement has a typical signal efficiency of about $80 \%$ while rejecting about $85 \%$ of the background. A tighter requirement in the $\bar{B}^{0} \rightarrow \bar{D}^{0} \bar{K}^{* 0}$ mode rejects $95 \%$ of the background and has a signal efficency of $55 \%$. The requirements on angle $\theta_{B}^{*}$ are $\left|\cos \theta_{B}^{*}\right|<0.75$ for $\bar{B}^{0} \rightarrow \bar{D}^{0} \bar{K}^{* 0}$, and $\left|\cos \theta_{B}^{*}\right|<0.85$ for all other decay modes.

In about $2-3 \%$ of the events more than one $B^{0}$ candidate with $m_{\mathrm{ES}}>5.2 \mathrm{GeV} / c^{2}$ satisfy the selection criteria. In the $D^{0} \bar{K}^{0}, D^{0} \bar{K}^{* 0}$, and $\bar{D}^{0} \bar{K}^{* 0}$ final states the candidate with the smallest $\Delta E$ is selected. In the $D^{* 0} K_{S}^{0}$ final state, we pick the candidate with the smallest $\chi^{2}$ computed from the measured value of $m\left(D^{0}\right)$ and $m\left(D^{* 0}\right)-m\left(D^{0}\right)$, their nominal values, and their resolutions in data.

The signal yield for each $B^{0}$ decay mode is determined with a binned maximum likelihood fit to the $m_{\mathrm{ES}}$ distribution for each $D^{0}$ decay mode. The distribution is modeled with a Gaussian for the signal and a threshold function for the combinatorial background. The mean and the r.m.s. resolution of the Gaussian are fixed to values measured in the $B^{+} \rightarrow \bar{D}^{0} \pi^{+}$calibration sample. The threshold function parameterizing the background is defined as $\mathcal{A}\left(m_{\mathrm{ES}}\right) \sim m_{\mathrm{ES}} \sqrt{1-x^{2}} \exp \{-\xi(1-$ $\left.\left.x^{2}\right)\right\}$ [10], where $x=2 m_{\mathrm{ES}} / \sqrt{s}$ and $\xi$ is a shape parameter.

The measured signal yields are summarized in Table 1 , and the $m_{\mathrm{ES}}$ distributions for the sums of all three $D^{0}$ decay modes are illustrated in Figure 2. The signal yields measured from the $\Delta E$ distribution, after removing the requirement on $\Delta E$ and selecting candidates with $5.273 \mathrm{GeV} / c^{2}<$ $m_{\mathrm{ES}}<5.288 \mathrm{GeV} / c^{2}$, are found to be in good agreement with the $m_{\mathrm{ES}}$ yields. In this case, for the small fraction of events with more than one selected candidates, we choose the one with $m_{\mathrm{ES}}$ closest to the $B$ mass. The $\Delta E$ distribution for the signal is modeled with a Gaussian and a second-order polynomial is used for the background. The $\Delta E$ distributions for the selected events are also shown in Figure 2. The combinatorial background in both $m_{\mathrm{ES}}$ and $\Delta E$ distributions are described well by events in the sidebands of the $D^{0}$ mass, which are shown as hatched histograms in Figure 2 . As a further cross check we examine the $K^{* 0}$ helicity angle $\theta_{h}$ of the $\bar{B}^{0} \rightarrow D^{0} \bar{K}^{* 0}$ candidates with 


\begin{tabular}{lcccc}
\hline$D^{0}$ channel & $N_{S}$ & $N^{\mathrm{pk}}$ & $\varepsilon_{\text {eff }}\left(10^{-3}\right)$ & $\mathcal{B}\left(10^{-5}\right)$ \\
\hline \multicolumn{5}{c}{$B \rightarrow D^{0} \overline{K^{0}}$} \\
\hline$K^{-} \pi^{+}$ & $18.9 \pm 5.6$ & $2.1 \pm 1.1$ & 2.85 & $4.8 \pm 1.6 \pm 0.5$ \\
$K^{-} \pi^{+} \pi^{0}$ & $18.0 \pm 6.3$ & $0.9 \pm 0.5$ & 2.20 & $6.3 \pm 2.3 \pm 0.7$ \\
$K^{-} \pi^{+} \pi^{-} \pi^{+}$ & $26.8 \pm 6.9$ & $0.0 \pm 1.1$ & 2.10 & $10.3 \pm 2.7 \pm 0.9$ \\
All & $64 \pm 11$ & $2.1 \pm 1.1$ & 7.15 & $6.2 \pm 1.2 \pm 0.4$ \\
\hline \multicolumn{5}{c}{$B \rightarrow D^{* 0} \bar{K}^{0}$} \\
\hline$K^{-} \pi^{+}$ & $3.1 \pm 2.0$ & $0.3 \pm 0.2$ & 0.80 \\
$K^{-} \pi^{+} \pi^{0}$ & $4.9 \pm 2.7$ & $0.2 \pm 0.2$ & 0.51 & $3.3 \pm 2.2 \pm 0.6$ \\
$K^{-} \pi^{+} \pi^{-} \pi^{+}$ & $3.2 \pm 2.0$ & $0.1 \pm 0.1$ & 0.34 & $8.6 \pm 5.5 \pm 1.0$ \\
All & $11.2 \pm 3.9$ & $0.8 \pm 0.4$ & 1.66 & $4.5 \pm 1.9 \pm 0.5$ \\
\hline \multicolumn{5}{c}{$\bar{B}^{0} \rightarrow D^{0} \bar{K}^{* 0}$} \\
\hline$K^{-} \pi^{+}$ & $17.7 \pm 5.2$ & $1.6 \pm 0.8$ & 2.34 \\
$K^{-} \pi^{+} \pi^{0}$ & $11.7 \pm 5.5$ & $1.0 \pm 0.5$ & 2.09 & $5.6 \pm 2.1 \pm 0.8$ \\
$K^{-} \pi^{+} \pi^{-} \pi^{+}$ & $14.9 \pm 5.4$ & $1.5 \pm 0.8$ & 2.10 & $6.7 \pm 2.7 \pm 1.0$ \\
All & $45.2 \pm 9.2$ & $4.4 \pm 2.2$ & 6.54 & $6.2 \pm 1.4 \pm 0.6$ \\
\hline \multicolumn{5}{c}{$\bar{B}^{0} \rightarrow \bar{D}^{0} \bar{K}^{* 0}$} \\
\hline All & $11.0 \pm 5.9$ & $5.5 \pm 4.5$ & 4.43 & $<4.190 \%$ C.L. \\
\hline
\end{tabular}

Table 1: Signal yield $N_{S}$, estimated peaking background $N^{\mathrm{pk}}$, effective signal efficiency $\varepsilon_{\text {eff }}$, and the measured branching fraction $\mathcal{B}$ for the $B \rightarrow D^{(*) 0} \bar{K}^{(*) 0}$ decays. The efficiency $\varepsilon_{\text {eff }}$ is defined as $\varepsilon \times \mathrm{BF}$, where $\varepsilon$ is the signal reconstruction efficiency and BF are the appropriate intermediate branching fractions for $D^{* 0}, D^{0}, K^{* 0}$, and $K^{0}$ decays to final states reconstructed in this analysis.

$5.273 \mathrm{GeV} / c^{2}<m_{\mathrm{ES}}<5.288 \mathrm{GeV} / c^{2}$ after removing the requirement $\left|\cos \theta_{h}\right|>0.4$. Figure 3 shows the distribution of $\cos \theta_{h}$ for these candidates, after subtracting the combinatorial background from the $D^{0}$ sidebands. We fit this spectrum with a sum of a flat component for the background and $\cos ^{2} \theta_{h}$ distribution for the signal, and find the fraction of the former to be consistent with zero as expected.

The measured signal yields $N_{S}$ include a small contribution from other $B$ decays that can be mis-reconstructed as signal events and accumulate near the $B$ mass. We have studied the contribution of such potential "peaking" backgrounds with large samples of simulated events, corresponding to typically between 100 and 1000 times the size of our data, for the following categories of decays: (1) $B^{0} \rightarrow \bar{D}^{0} \rho^{0}, \rho^{0} \rightarrow \pi^{+} \pi^{-}$decays, where one of the two pions is mis-identified as a charged kaon; (2) the $B^{0} \rightarrow D^{-} \pi^{+}$decays, followed by the Cabibbo-suppressed decays $D^{-} \rightarrow K^{(*) 0} K^{-}$ and $B^{0} \rightarrow D^{-} K^{+}, D^{-} \rightarrow K^{(*) 0} \pi^{-}$reconstructed in the $D^{0}\left(K^{-} \pi^{+}\right) K^{(*) 0}$ final states; (3) charmless $B^{0} \rightarrow K^{+} \pi^{-} K_{S}^{0}(n \pi)$ where the $K^{+}$and $\pi^{-}$are wrongly combined to form a $\bar{D}^{0} \rightarrow K^{+} \pi^{-}$ candidate; (4) $B^{0} \rightarrow D^{* 0} \bar{K}^{(*) 0}, D^{* 0} \rightarrow D^{0} \gamma$ candidates, where a low-energy photon $\gamma$ is not reconstructed; (5) the decays $B^{+} \rightarrow \bar{D}^{* 0} K^{+}, \bar{D}^{* 0} \rightarrow \bar{D}^{0} \pi^{0} / \gamma, B^{+} \rightarrow \bar{D}^{0} K^{*+}, K^{*+} \rightarrow K^{+} \pi^{0}, K_{S}^{0} \pi^{+}$, and $B^{0} \rightarrow D^{*-} K^{+}, D^{*-} \rightarrow \bar{D}^{0} \pi^{-}$, where a low-energy $\pi^{0}$ or photon is replaced by a random lowmomentum charged track. The contribution of category (1) is found to be less than 0.01 events and is hence neglected. The contribution of category (2) is also negligible in all modes, except for $B \rightarrow D^{0} \bar{K}^{0}, D^{0} \rightarrow K^{-} \pi^{+}$. We eliminate $87 \%$ of these events by requiring the invariant masses $m\left(K_{S}^{0} K^{-}\right)$and $m\left(K_{S}^{0} \pi^{+}\right)$to be more than $20 \mathrm{MeV} / c^{2}$ away from the nominal $D^{+}$mass. The $m_{\mathrm{ES}}$ spectrum of the remaining background events in this category and in categories (3)-(5) shows a 
broad enhancement near the $B$ mass. The contributions of these events to the signal yields are measured by performing a Monte Carlo study and are summarized in Table 1 . We assign a $50 \%$ systematic error to the estimated peaking background contribution due to the uncertainty on the branching fractions of some of these $B$ decays. In the decay $\bar{B}^{0} \rightarrow \bar{D}^{0} \bar{K}^{* 0}$, the charge correlation used in the selection removes all contributions from known $B$ decays included in our simulation. We estimate the peaking background for this decay mode from the $D^{0}$ sidebands to be $5.5 \pm 4.5$ events.

The significance of the signal yields is determined by taking into account the sum $N_{B}$ of the combinatorial and peaking backgrounds and its uncertainty $\delta N_{B}$. We generate one million experiments where the expected number of background $N_{B}^{i}$ for the $i$-th experiment is extracted from a Gaussian with mean $N_{B}$ and width $\delta N_{B}$. Assuming a Poisson distribution for the background events, the significance is determined from the fraction of experiments with $N_{B}^{i}>N_{\text {obs }}$ with $N_{\text {obs }}$ being the number of candidates in data with $5.273 \mathrm{GeV} / c^{2}<m_{\mathrm{ES}}<5.288 \mathrm{GeV} / c^{2}$. The $B \rightarrow D^{* 0} \bar{K}^{0}$ signal has a significance of $3.3 \sigma$ and is the first evidence for this decay mode. The significance of the $\bar{B}^{0} \rightarrow D^{0} \bar{K}^{* 0}$ and $\bar{B}^{0} \rightarrow \bar{D}^{0} \bar{K}^{* 0}$ are, respectively, $4.8 \sigma$ and $2.0 \sigma$. For the $D^{0} \bar{K}^{0}$ final state, where the number of observed events is large, we estimate the signal significance to be $5.8 \sigma$ from the measured signal yield and its uncertainty.

The systematic uncertainties for the branching fractions are reported in Table 1 and include contributions from estimated peaking background (3-11\%), fit parameters $(2-8 \%), D^{(*) 0}$ branching fraction $(2.4-6.9 \%), \pi^{0}$ reconstruction efficiency (2.5\% per photon), charged-track reconstruction efficiency ( $0.8 \%$ per track), Monte Carlo statistics (1-4\%), efficiency correction factors (1-4\%), kaon identification (2\% per kaon), $K_{S}^{0}$ reconstruction efficiency (1.6\%), and number of $B \bar{B}$ events $(1.1 \%)$.

The branching fraction $\mathcal{B}$ of each $B^{0}$ decay mode is computed as the weighted average of the branching fractions $B_{j}$ in each $D^{0}$ channel $D_{j}=\left\{K^{-} \pi^{+}, K^{-} \pi^{+} \pi^{-} \pi^{+}, K^{-} \pi^{+} \pi^{0}\right\}$, computed as

$$
\mathcal{B}_{j}=\frac{N_{S_{j}}-N_{j}^{\mathrm{pk}}}{N_{B \bar{B}} \times \mathcal{B}_{D_{j}} \times \mathcal{B}_{K} \times \varepsilon_{j}}
$$

where $N_{S_{j}}$ is the signal yield from the $m_{\mathrm{ES}}$ fit, $N_{j}^{\mathrm{pk}}$ is the estimated peaking background from Table $1, N_{B \bar{B}}$ is the total number of $\Upsilon(4 S) \rightarrow B \bar{B}$ events, $\mathcal{B}_{D_{j}}$ is the branching fraction $\mathcal{B}\left(D^{0} \rightarrow\right.$ $\left.D_{j}\right)$ in $B \rightarrow D^{0} \bar{K}^{(*) 0}$ and $\mathcal{B}\left(D^{* 0} \rightarrow D^{0} \pi^{0}\right) \times \mathcal{B}\left(D^{0} \rightarrow D_{j}\right)$ in $B \rightarrow D^{* 0} \bar{K}^{0}, \mathcal{B}_{K}$ is the known $K^{0} \rightarrow K_{S}^{0} \rightarrow \pi^{+} \pi^{-}\left(K^{* 0} \rightarrow K^{+} \pi^{-}\right)$branching fraction in $B \rightarrow D^{(*) 0} \bar{K}^{0}\left(\bar{K}^{* 0}\right)$, and $\varepsilon_{j}$ is the signal reconstruction efficiency. We assume $\mathcal{B}\left(\Upsilon(4 S) \rightarrow B^{0} \bar{B}^{0}\right)=0.5$.

We measure

$$
\begin{aligned}
\mathcal{B}\left(B \rightarrow D^{0} \bar{K}^{0}\right) & =(6.2 \pm 1.2 \pm 0.4) \times 10^{-5} \\
\mathcal{B}\left(B \rightarrow D^{* 0} \bar{K}^{0}\right) & =(4.5 \pm 1.9 \pm 0.5) \times 10^{-5} \\
\mathcal{B}\left(\bar{B}^{0} \rightarrow D^{0} \bar{K}^{* 0}\right) & =(6.2 \pm 1.4 \pm 0.6) \times 10^{-5} \\
\mathcal{B}\left(\bar{B}^{0} \rightarrow \bar{D}^{0} \bar{K}^{* 0}\right) & =(1.1 \pm 1.1 \pm 1.2) \times 10^{-5}
\end{aligned}
$$

where the uncertainties are, respectively, statistical and systematic. For the decay $\bar{B}^{0} \rightarrow \bar{D}^{0} \bar{K}^{* 0}$ we use the Bayesian method to compute the upper limit $N_{U L}$ on the observed number of events at $90 \%$ confidence level as $\int_{0}^{N_{U L}} \mathcal{L}(N) d N=0.9$, where $\mathcal{L}(N)$ is the binned maximum likelihood function from the fit to the $m_{\mathrm{ES}}$ distribution. We assume a flat prior probability density function for $\mathcal{B}>0$. After accounting for the systematic uncertainties we obtain at $90 \%$ C.L. $\mathcal{B}\left(\bar{B}^{0} \rightarrow\right.$ $\left.\bar{D}^{0} \bar{K}^{* 0}\right)<4.1 \times 10^{-5}$. 
In summary, we have presented evidence for the decay $\bar{B}^{0} \rightarrow D^{* 0} \bar{K}^{0}$ as well as new measurements of the branching fractions for the decays $\bar{B}^{0} \rightarrow D^{0} \bar{K}^{0}$ and $D^{0} \bar{K}^{* 0}$. Our measurements are in agreement with the expectation derived from Ref. [5] and with previous measurements [11]. We use the central value of our measurement for $\mathcal{B}\left(\bar{B}^{0} \rightarrow \bar{D}^{0} \bar{K}^{* 0}\right)$ and obtain $r<0.8$ at the $90 \%$ C.L. from a central value of $r=0.4 \pm 0.2$ (stat.) \pm 0.2 (syst.). The main contribution to the systematic uncertainty is from the estimated peaking background since most systematic uncertainties on the branching fractions cancel in the ratio.

We are grateful for the excellent luminosity and machine conditions provided by our PEP-II colleagues, and for the substantial dedicated effort from the computing organizations that support $B A B A R$. The collaborating institutions wish to thank SLAC for its support and kind hospitality. This work is supported by DOE and NSF (USA), NSERC (Canada), IHEP (China), CEA and CNRS-IN2P3 (France), BMBF and DFG (Germany), INFN (Italy), FOM (The Netherlands), NFR (Norway), MIST (Russia), and PPARC (United Kingdom). Individuals have received support from CONACyT (Mexico), A. P. Sloan Foundation, Research Corporation, and Alexander von Humboldt Foundation.

\section{References}

[1] BABAR Collaboration, B. Aubert et al., Phys. Rev. Lett. 87, 091801 (2001); Belle Collaboration, K. Abe et al., Phys. Rev. Lett. 87, 091802 (2001).

[2] BABAR Collaboration, B. Aubert et al., Phys. Rev. Lett. 89, 201802 (2002); Belle Collaboration, K. Abe et al., Phys. Rev. D 66, 071102(R) (2002).

[3] N. Cabibbo, Phys. Rev. Lett. 10, 531 (1963); M. Kobayashi and T. Maskawa, Prog. Theor. Phys. 49, 652 (1973).

[4] M. Gronau and D. London, Phys. Lett. B 253, 483 (1991); D. Atwood, I. Dunietz, and A. Soni, Phys. Rev. Lett. 78, 3257 (1997); B. Kayser and D. London, Phys. Rev. D 61, 116013 (2000); A. I. Sanda, hep-ph/0108031.

[5] CLEO Collaboration, T. E. Coan et al.,Phys. Rev. Lett. 88, 062001 (2002); Belle Collaboration, K. Abe et al., Phys. Rev. Lett. 88, 052002 (2002); Belle Collaboration, A. Satpathy et al., Phys. Lett. B 553, 159 (2003); BABAR Collaboration, B. Aubert et al., Phys. Rev. D 69, 032004 (2004).

[6] BABAR Collaboration, B. Aubert et al., Nucl. Instrum. Methods A479, 1 (2002).

[7] Particle Data Group, S. Eidelman et al., Phys. Lett. B 592, 1 (2004).

[8] CLEO Collaboration, S. Kopp et al., Phys. Rev. D 63, 092001 (2001).

[9] R. A. Fisher, Annals Eugen. 7, 179 (1936).

[10] ARGUS Collaboration, Z. Phys. C48, 543 (1990).

[11] Belle Collaboration, P. Krokovny et al., Phys. Rev. Lett. 90, 141802 (2003). 

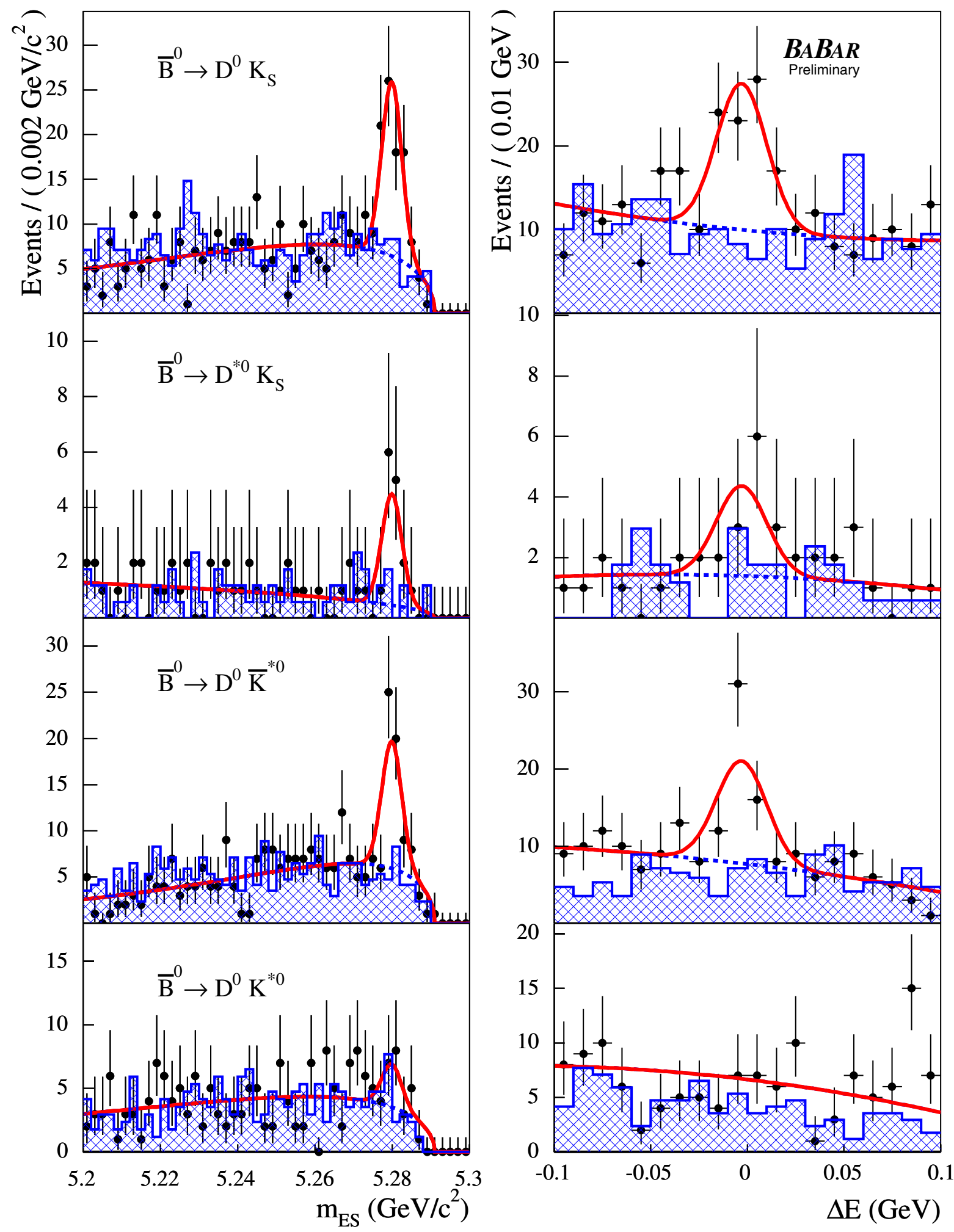

Figure 2: Distribution of $m_{\mathrm{ES}}$ (left) and $\Delta E$ (right) for $B \rightarrow D^{(*) 0} \bar{K}^{0}$ and $\bar{B}^{0} \rightarrow D^{0} \bar{K}^{* 0}$ and $\bar{D}^{0} \bar{K}^{* 0}$ candidates. The points are the data, the curve is the result of the fit, and the hatched histogram is the distribution of candidates in $D^{0}$ sidebands. 


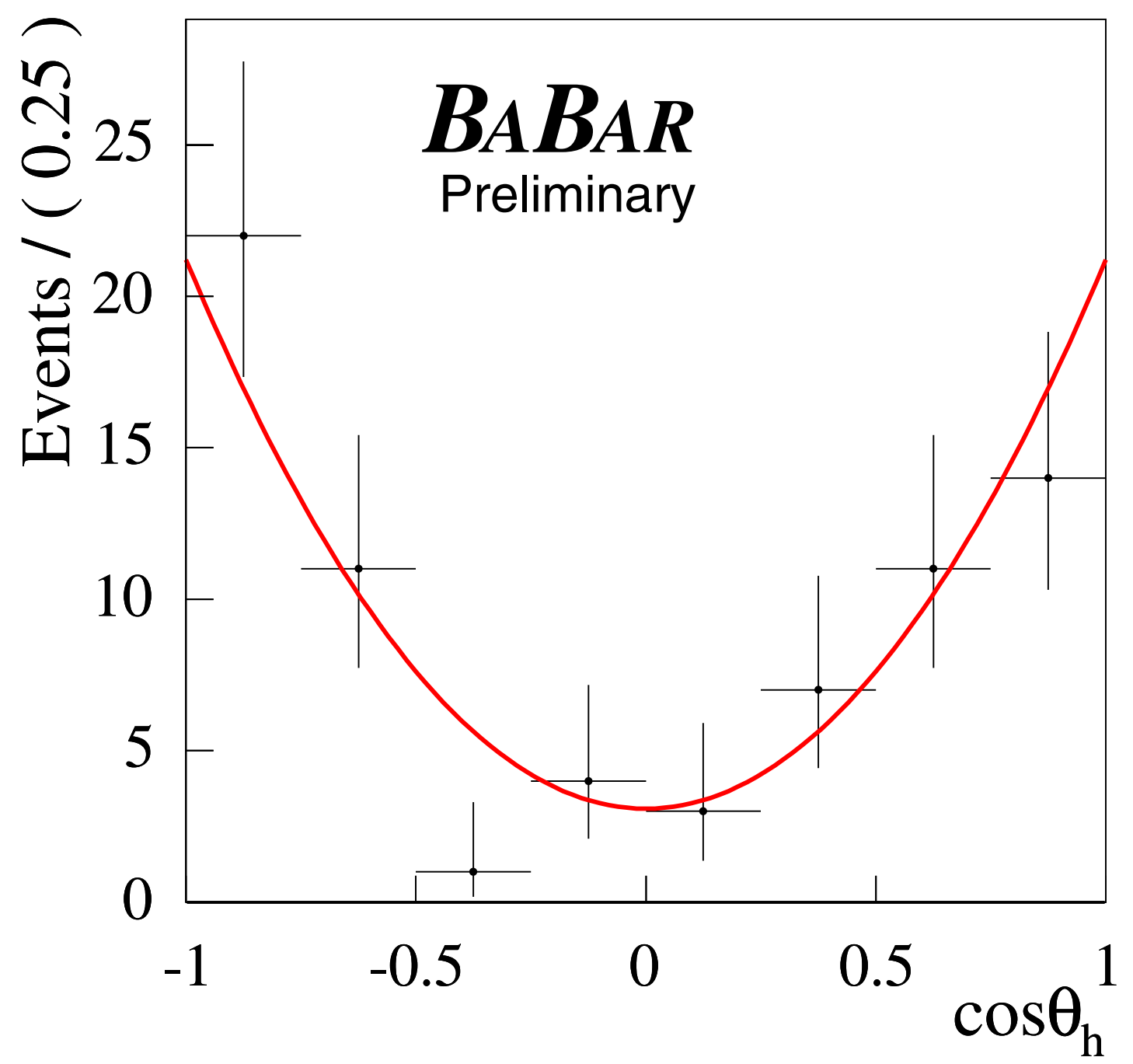

Figure 3: Distribution of $\cos \theta_{h}$ for selected $\bar{B}^{0} \rightarrow D^{0} \bar{K}^{* 0}$ candidates. The points are data, after subtracting the combinatorial background, and the curve is the result of a fit, which is consistent with the expected $\cos ^{2} \theta_{h}$ distribution. 